Research Article

\title{
Significance of Nonsimilar Numerical Simulations in Forced Convection from Stretching Cylinder Subjected to External Magnetized Flow of Sisko Fluid
}

\author{
Jifeng Cui ${ }^{D},{ }^{1}$ Umer Farooq $\mathbb{D}^{2},{ }^{2}$ Ahmed Jan, ${ }^{2}$ Murtada K. Elbashir $\left(\mathbb{D},{ }^{3,4}\right.$ \\ Waseem Asghar Khan $\left(D,{ }^{5}\right.$ Mogtaba Mohammed, ${ }^{5}$ Ziyad Ali Alhussain, ${ }^{5}$ \\ and Jamshaid UI Rahman ${ }^{6}$ \\ ${ }^{1}$ College of Science, Inner Mongolia University of Technology, Hohhot 010051, China \\ ${ }^{2}$ Department of Mathematics, COMSATS University Islamabad, Park Road Chak Shahzad, Islamabad 44000, Pakistan \\ ${ }^{3}$ Faculty of Mathematical and Computer Sciences, University of Gezira, Wad Madani 11123, Sudan \\ ${ }^{4}$ College of Computer and Information Sciences, Jouf University, Sakaka 72441, Saudi Arabia \\ ${ }^{5}$ Department of Mathematics, Faculty of Sciences AlZulfi, Majmaah University, Majmaah 11952, Saudi Arabia \\ ${ }^{6}$ Abdus Salam School of Mathematical Sciences, GCU, Lahore 54000, Pakistan
}

Correspondence should be addressed to Jifeng Cui; cjf@imut.edu.cn and Murtada K. Elbashir; mkelfaki@ju.edu.sa

Received 18 July 2021; Revised 18 August 2021; Accepted 31 October 2021; Published 24 November 2021

Academic Editor: Qingkai Zhao

Copyright @ 2021 Jifeng Cui et al. This is an open access article distributed under the Creative Commons Attribution License, which permits unrestricted use, distribution, and reproduction in any medium, provided the original work is properly cited.

\begin{abstract}
The practice of flowing effort is participating in various industries especially in nutrition productions all around the world. These fluids practices are utilized extensively in nutrition handling productions by making use of sticky liquids to produce valuable food manufactured goods in bulk. Nevertheless, such productions ought to guarantee that involved equipment such as pipelines are maintained clean as well as are cleared out for the efficient movement of fluids. The nonsimilar characteristics of involuntary convection from circular cylinder stretching in the axial direction subjected to an external flow of Sisko fluid characterized by the freely growing boundary layers (BL) are presented in this research. A circular cylinder is submerged in a stationary fluid. The axial stretching of the cylinder causes external fluid flow. The magnetic force of strength " $B_{0}$ " is enforced in the transverse direction. Because of the fluid's high viscosity, frictional heating due to viscous dissipation is quite significant. The flow is three dimensional but with no circumferential variations. The governing equations for axisymmetric flow that include the mass balance, $x$-momentum, and heat equation are modeled through conservation laws. The dimensionless system is developed by employing appropriate nonsimilar transformations. The numerical analyses are presented by adapting local nonsimilarity via finite-difference (FDM)-based MATLAB algorithm bvp4c. The characteristics of dimensionless numbers are determined by graphs that are plotted on momentum and heat equations. The nonsimilar simulations have been compared with the existing local similar solutions. Fluid velocity is increased as the material and curvature parameters are increased, resulting in improved heat transfer. The deviation in skin friction and local Nusselt number against the various dimensionless numbers is also analyzed.
\end{abstract}

\section{Introduction}

The evaluation of non-Newtonian fluids won plenty of attention between the scholars in past because of its large-scale applications in metal spinning, polymer extrusion, and fabrication fields. Since a massive number of non-Newtonian fluids exist, therefore, a bunch of fluid simulations is planned to examine the material characteristics of these fluids. As the power law or generalized Newtonian fluid model is the best relevant model to portend the mindset of non-Newtonian fluids. The procedure of liquid by means of transport in food stuff productions throughout the globe is of fantastic importance. Extremely nutrition handling productions make use of sticky liquids to produce valuable food manufactured goods in majority. Nevertheless, such productions ought to guarantee that involved kit such as pipelines are maintained clean as well 
as are cleared out for the efficient movement of liquids. The liquids are injected in network which results in friction over the wall up of the tube $[1,2]$. Movement qualities are the basis of the mass shifts taking place in food stuff productions, e.g., honey, which is utilized in medication and diets due to its biological produce found from nectar, and patients of all age group use these stuffs on regular basis in all seasons. Furthermore, vitamins, high value minerals, and proteins are also components of honey. Consequently, the flow performances of honey at all times have a fascinating subject matter. Honey is also widely utilized as a food preservative and food component as well. The honey found from beehives and scrutinizes has honeycomb, pollen, and other unattractive ingredients. The ejection of these substances is crucial for a generally nice value product alongside with enhancing shelf life. In nutrition industries, honey goes through various procedures, prior to being jam-packed into cans and containers, such as central heating and purification to remove additional waste materials. Furthermore, the humidity substance would be monitored to reduce the risks of fermentation [1-3].

When the shear rate becomes very low or high these fluids stop to inspect the flow characteristics, the Sisko fluid model is one of the numerous non-Newtonian fluid models, and it has vast industrial, mechanical, and automobile engineering applications such as lubricating oil, food products, and cementitious slurry. It can be used as an antiwear agent, extreme pressure agent, and corrosion inhibitor and inhibit seizure under high loads and temperatures. In recent years, scholars examined Sisko fluid with different physical hypotheses over various geometries. Sisko [4] worked on greases that depict non-Newtonian behavior in nature having high viscosity with low shear rate and vice versa. Alyiuldiz et al. [5] reviewed the thin film Sisko fluid flow on the top of a moving belt and acquired analytical solutions by employing the homotopy method (HAM). Nadeem and Akbar [6] examined the peristaltic Sisko fluid flow in the uniform inclined duct through. Khan et al. [7] modeled nonlinear equations of incompressible Sisko fluid through a pipe and obtained both approximate and numerical results by using HAM and FDM, respectively. Munir et al. [8] studied the characteristics of 3D, steady flow of Sisko fluid which is triggered due to bi-directional stretching. Malik et al. [9] implored thermal transport of the flow of Sisko fluid across a cylinder. Casson 3D fluid flow subjected to magnetohydrodynamic (MHD) past on top of a permeable surface was numerically viewed by Nadeem et al. [10]. Mustafa and Khan [11] presented MHD drift of Casson nanofluid on top of a nonlinearly expanding cylinder. Farooq et al. [12] inspected the MHD flow of non-Newtonian fluid across a stretching surface. Rashidi et al. [13] inspected numerically the impacts of slip factor, magnetic interaction, and temperature gradient across a rotating disk. Malik et al. [14] analyzed the thermal transport of fluid flow across a cylinder with the consequences of magnetic force. The utilization of numerical procedures in hydraulics networks and various engineering applications can be seen in $[15,16]$.

During the experiments, "the fluids are considered in motion, during motion fluid particles, which transforms some of their kinetic energy (K. E) into thermal heat due to viscosity, and the process is irreversible," and this process is mentioned as viscous dissipation. Brikman [17] investigated viscous dissipation and its properties. He discussed the temperature distribution of the Newtonian fluid in a linear round duct and illuminated the results that were produced in the locked region. Abd El-Aziz [18] measured the outcomes of the viscous dissipation for Sisko fluid flow along with a semiinfinite stretching sheet. Saleem and Nadeem [19] examined the viscous dissipation influences over a vertically rotating cone by applying HAM. Moreover, compilations of publications on viscous dissipation can be found in [20-26].

Mathematical models of physical procedures in fields of wave dynamics, fluid mechanics, biological kinetics, diffusion, and transportation problems are driven by nonlinear partial differential equations (PDEs). The governing PDEs of a BL fluid flow are transformed into dimensionless PDEs. The considerable mathematical difficulty is associated with the solutions of these $\mathrm{BL}$ equations of fluid flow mainly because of their nonlinearity. Several approximate analytical and numerical methods have been established to tackle these equations. Although the local nonsimilar method (LNSM) presented by Sparrow and Yu [27] is more accurate and efficient than other techniques, it is only an approximate method since PDEs are reduced to ODEs after some degree of approximation truncation. Lately, several authors have conducted extensive research on nonsimilar flows primarily using the approach of LNSM [28-34].

In this study, the magnetized flow of Sisko fluid over a circular cylinder stretching in the axial direction is investigated. The nonsimilar dimensionless system is numerically simulated by employing LNSM up to the second level of truncation via bvp4c. The drawback of local similarity method (LSM) is the governing PDEs that were obtained after the transformation has similar terms. To overcome this drawback, Sparrow et al. [35] used the method of local nonsimilarity. Impacts of the emerging dimensionless parameters, e.g., material parameter, curvature parameter, Prandtl number, and magnetic parameter, are studied on the important numbers in thermal transport analysis such as frictional drag and local Nusselt number and profiles of velocity and temperature through tables and graphs. Practical implications of this study can be found in [36-41].

\section{Convection Equations for Transport Analysis}

Consider a circular cylinder, which is submerged in a stationary fluid. The forced external flow is initiated due to the cylinder expansion in the axial direction with the velocity $U(x)=c x$, where $c$ is time constant. The flow is steady, incompressible, and axisymmetric. The transverse magnetic field with strength $B_{o}$ is imposed in $r$-direction. Induced magnetic and electric fields are ignored. Frictional heating due to viscous dissipation is significant because of the high viscosity of the fluid. Therefore, the viscous shear stress impact is incorporated in the energy equation.

Problem geometry is indicated in Figure 1.

Using the assumptions stated above, the convection equations are given by [42] 


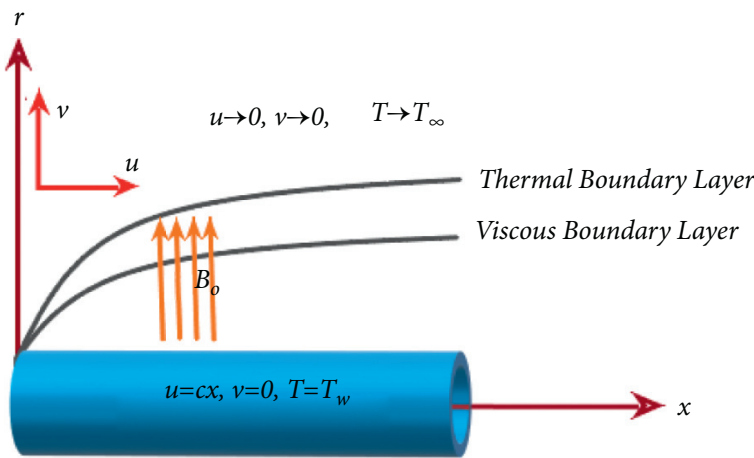

FIGURE 1: Schematic for stretching cylinder submerged in a stationary fluid.

$$
\begin{aligned}
\frac{\partial(r u)}{\partial x}+\frac{\partial(r v)}{\partial r} & =0 \\
u \frac{\partial u}{\partial x}+v \frac{\partial u}{\partial r} & =\frac{a}{r \rho} \frac{\partial}{\partial r}\left(r \frac{\partial u}{\partial r}\right)-\frac{b}{r \rho}\left(-\frac{\partial u}{\partial r}\right)^{n}+\frac{n b}{\rho}\left(-\frac{\partial u}{\partial r}\right)^{n-1} \frac{\partial^{2} u}{\partial r^{2}}-\frac{\sigma B_{0}^{2}}{\rho} u \\
u \frac{\partial T}{\partial x}+v \frac{\partial T}{\partial r} & =\frac{\alpha}{\rho} \frac{\partial}{\partial r}\left(r \frac{\partial T}{\partial r}\right)-\frac{a}{\rho C_{p}}\left(\frac{\partial u}{\partial r}\right)^{2}+\frac{b}{\rho C_{p}}\left(-\frac{\partial u}{\partial r}\right)^{n+1},
\end{aligned}
$$

under boundary conditions

$$
\begin{aligned}
& u=U(x), v=0, T=T_{w} \text { at } r \longrightarrow r_{o}, \\
& u \longrightarrow 0, v \longrightarrow 0, T \longrightarrow T_{\infty} \text { at } r \longrightarrow \infty .
\end{aligned}
$$

where $u-v$ are the components of fluid velocity along the $x$ axis and $r$-direction. The material constants at high shear rate viscosity are $a$ and $\sigma$ which represent an electrical conductivity, power-law index denoted by $n$, and $b$ is the consistency index. The magnetic field $B_{o}$, the fluid density is $\rho, \alpha$ indicates the thermal diffusivity, and $C_{p}, T_{w}$, and $T_{\infty}$ are the specific heat, wall, and ambient temperature.

To develop a nonsimilar flow, introducing a new variable $\xi(x)$ and $\eta(x, y)$ such that

$$
\begin{aligned}
\xi & =\frac{x}{l}, \\
\eta & =\frac{r^{2}-r_{o}^{2}}{2 x r_{o}} \operatorname{Re}_{b}^{1 /(n+1)}, \\
u & =x c \frac{\partial f}{\partial \eta}(\xi, \eta), \\
v & =-\frac{x c r_{o}}{r} \operatorname{Re}_{b}^{1 /(n+1)}\left(2 f(\xi, \eta)+\eta \frac{\partial f}{\partial \eta}(\xi, \eta)+\xi \frac{\partial f}{\partial \xi}(\xi, \eta)\right), \\
\theta(\xi, \eta) & =\frac{\left(T-T_{\infty}\right)}{\left(T_{w}-T_{\infty}\right)}, \\
\operatorname{Re}_{b} & =\frac{\rho l^{2} c^{2-n}}{b} .
\end{aligned}
$$

Using (5) in (1)-(4), we get the following dimensionless nonsimilar system: 


$$
\begin{gathered}
A \xi^{-2}(1+2 \gamma \eta \xi) \frac{\partial^{3} f}{\partial \eta^{3}}+2 A \gamma \xi^{-1} \frac{\partial^{2} f}{\partial \eta^{2}}-\gamma \xi^{-1}(1+n)(1+2 \gamma \eta \xi)^{(n-1) / 2}\left(-\frac{\partial^{2} f}{\partial \eta^{2}}\right)^{n}-\left(\frac{\partial f}{\partial \eta}\right)^{2} \\
+2 f \frac{\partial^{2} f}{\partial \eta^{2}}-M \frac{\partial f}{\partial \eta}+n \xi^{-2}(1+2 \gamma \eta \xi)^{(n+1) / 2} \frac{\partial^{3} f}{\partial \eta^{3}}\left(-\frac{\partial^{2} f}{\partial \eta^{2}}\right)^{n-1}=\xi\left(\frac{\partial f}{\partial \eta} \frac{\partial^{2} f}{\partial \xi}-\frac{\partial f}{\partial \xi} \frac{\partial^{2} f}{\partial \eta^{2}}\right) \\
\xi^{-2}(1+2 \gamma \eta \xi) \frac{\partial^{2} \theta}{\partial \eta^{2}}+A \operatorname{Pr} E c(1+2 \gamma \eta \xi)\left(\frac{\partial^{2} f}{\partial \eta^{2}}\right)^{2}+E c \operatorname{Pr}(1+2 \gamma \eta \xi)^{n+1 / 2} \\
\left(-\frac{\partial^{2} f}{\partial \eta^{2}}\right)^{n+1}+2 \gamma \xi^{-1} \frac{\partial \theta}{\partial \eta}+2 \operatorname{Pr} f \frac{\partial \theta}{\partial \eta}=\operatorname{Pr} \xi\left(\frac{\partial f}{\partial \eta} \frac{\partial \theta}{\partial \xi}-\frac{\partial f}{\partial \xi} \frac{\partial \theta}{\partial \eta}\right) .
\end{gathered}
$$

Nonsimilar boundary conditions are

$$
\begin{aligned}
\frac{\partial f(\xi, 0)}{\partial \eta} & =1, \\
2 f(\xi, 0)+\xi \frac{\partial f(\xi, 0)}{\partial \xi} & =0, \\
\theta(\xi, 0) & =1, \\
\frac{\partial f(\xi, \infty)}{\partial \eta} & =0, \\
\theta(\xi, \infty) & =0,
\end{aligned}
$$

where curvature parameter $\gamma=\left(1 / r_{o}\right) \mathrm{Re}_{b}^{-1 / n+1}$, magnetic number $M=\left(\sigma B_{o}^{2} / \rho c\right)$, Reynold number $\operatorname{Re}_{a}=\left(\rho l^{2} c / a\right)$, material parameter $A=\left(\mathrm{Re}_{b}^{-2 / n+1} / \mathrm{Re}_{a}\right)$, Eckert number $\mathrm{Ec}=\left(c^{2} l^{2} / C_{p}\left(T_{w}-T_{\infty}\right)\right), \quad$ and Prandtl number $\operatorname{Pr}=\left(c l^{2} / \alpha\right) \mathrm{Re}_{b}^{-2 / n+1}$ are the dimensionless quantities. are

Surface friction coefficient $C_{f}$ and Nusselt $\mathrm{Nu}_{x}$ numbers

$$
\begin{aligned}
C_{f} & =\frac{\tau_{w}}{(1 / 2) \rho U^{2}}, \\
\mathrm{Nu} & =\frac{x q_{w}}{K\left(T_{w}-T_{\infty}\right)},
\end{aligned}
$$

where $\tau_{w}$ surface shear stress, $q_{w}$ is the surface flux, and

$$
\begin{aligned}
\tau_{w} & =a\left(\frac{\partial u}{\partial r}\right)_{r=r_{o}}-b\left(-\frac{\partial u}{\partial r}\right)_{r=r_{o}}^{n}, \\
q_{w} & =-K\left(\frac{\partial T}{\partial y}\right)_{r=r_{o}} .
\end{aligned}
$$

Equations (9) and (10) in dimensionless forms are

$$
\begin{aligned}
\frac{1}{2} \operatorname{Re}_{b}^{1 / n+1} C_{f} & =\xi^{-2}\left(A f^{\prime \prime}(0)-\left(-f^{\prime \prime}(0)\right)^{n}\right), \\
\operatorname{Re}_{b}^{-(-1 / n+1)} \mathrm{Nu} & =-\theta^{\prime}(\xi, 0) .
\end{aligned}
$$

\section{Local Nonsimilarity Method}

Assuming $\xi<<1$ and the right-hand side of equations (6) and (7), $\xi(\partial(.) / \partial \xi)$ equal to zero. Therefore, the equations of local similarity are

$$
\begin{aligned}
f^{\prime \prime \prime} & =\frac{-2 A \gamma \xi^{-1} f^{\prime \prime}+f^{\prime 2}-2 f^{\prime \prime} f+M f^{\prime}+(1+n) \gamma \xi^{-1}(1+2 \gamma \eta \xi)^{n-1 / 2}\left(-f^{\prime \prime}\right)^{n}}{A \xi^{-2}(1+2 \gamma \eta \xi)+n \xi^{-2}(1+2 \gamma \eta \xi)^{n+1 / 2}\left(-f^{\prime \prime}\right)^{n-1}}, \\
\theta^{\prime \prime} & =\frac{-2 \gamma \xi^{-1} \theta^{\prime}-2 \operatorname{Pr} f \theta^{\prime}-A \operatorname{PrEc}(1+2 \gamma \eta \xi) f^{\prime \prime 2}-\operatorname{EcPr}(1+2 \gamma \eta \xi)^{n+1 / 2}\left(-f^{\prime \prime}\right)^{n+1}}{\xi^{-2}(1+2 \gamma \eta \xi)} .
\end{aligned}
$$

Under boundary conditions, 
TABLE 1: Skin friction values' comparison for diverse curvature parameter and $M=0$ and $A=0$, for $n=1$.

\begin{tabular}{lccc}
\hline$\gamma$ & $\begin{array}{c}\text { Malik et al. [42] } \\
\text { Local similar }\end{array}$ & $\begin{array}{c}\text { Rangi and Ahmad [43] } \\
\text { Local similar }\end{array}$ & $\begin{array}{c}\text { Present study } \\
\text { Nonsimilar }\end{array}$ \\
\hline 0 & -1.0007 & -1 & -0.9813 \\
0.25 & -1.0950 & -1.0944 & -1.0799 \\
0.5 & -1.1899 & -1.1887 & -1.1694 \\
0.75 & -1.2835 & -1.2818 & -1.2506 \\
1 & -1.4585 & -1.4593 & -1.3240 \\
\hline
\end{tabular}

$$
\begin{aligned}
f^{\prime}(\xi, 0) & =1, \\
f(\xi, 0) & =0, \\
\theta(\xi, 0) & =1, \\
\theta(\xi, \infty) & =0, \\
f^{\prime}(\xi, \infty) & =0 .
\end{aligned}
$$

To obtain $2^{\text {nd }}$ level truncation, differentiate equations (6) and (7) with respect $\xi$ and introduce new functions $g(\xi, \eta)=$ $(\partial f(\xi, \eta) / \partial \xi)$ and $h(\xi, \eta)=(\partial \theta(\xi, \eta) / \partial \xi)$ and put the derivatives of the introducing function with $\xi$ equal to zero as $(g(\xi, \eta) / \partial \xi)=0,(h(\xi, \eta) / \partial \xi) 0$. The transformed equations are

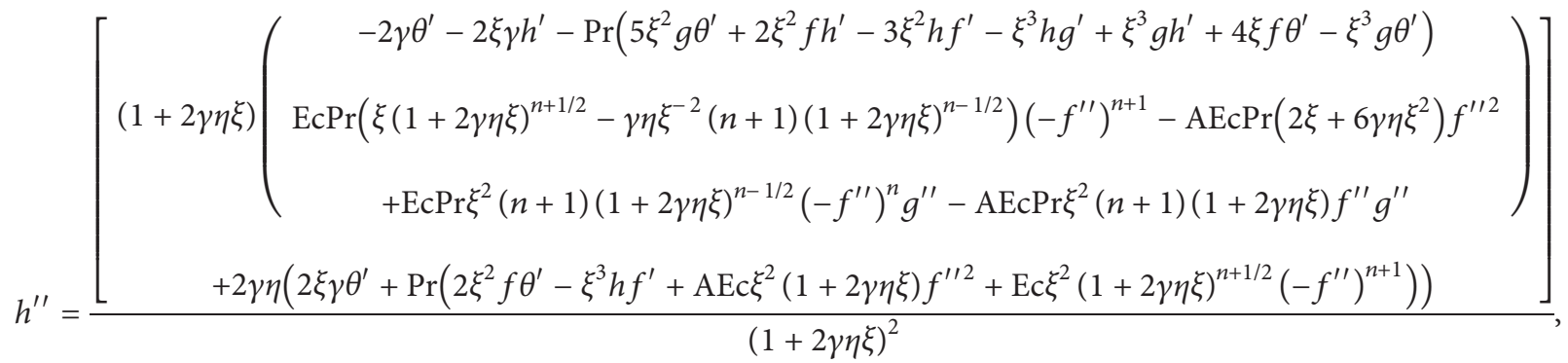

subjected to the conditions

$$
\begin{array}{r}
g^{\prime}(0)=0, \\
g(0)=0, \\
g^{\prime}(\infty)=0, \\
h(0)=0, \\
h(\infty)=0 .
\end{array}
$$

\section{Forced Convection Analysis}

The behavior of various dimensionless numbers is implored to study the variations in the thermal transport analysis. The significance of the nonsimilar modeling and its simulations are compared, and results are presented in Tables 1 and 2. Table 1 is the comparison with the existing works of Malik et al. [42] and Rangi and Ahmad [43]. Results of skin friction for varying the curvature parameter values are shown in Table 1. Table 2 reveals the difference between the current results with the published articles of Malik et al. [42] and Akbar et al. [44] for several values of magnetic number. Table 3 describes the impact of increasing $\gamma, A$, and $M$ on frictional coefficient. It is noticed that friction increases by enhancing numerical values of $\gamma, A$, and $M$. Table 4 illustrates that increasing $\gamma$ and $\operatorname{Pr}$ increases the rate of thermal transport; however, the impact of increasing Ec is to decline the rate of heat transfer. Figure 2 describes that increase in magnetic force boosts the Lorentz force which resist the fluid flow. Thus, a rise in the Lorentz force reduces the velocity field.

Curvature parameter behavior against velocity profile is portrayed in Figure 3. The curvature parameter is the ratio of boundary layer thickness to the cylinder's radius. The increasing curvature curtails the cylinder's diameter. The smaller radius implies that the contact surface of the cylinder is less; therefore, it will provide less struggle to fluid flow. As a result, the fluid accelerates, and it is determined that the flow escalates by boosting the curvature parameter. In Figure 4 , the property of $A$ on the velocity field is examined. The fluid velocities increase for significant values of the material parameter; physically, it retains because when the material parameter has significant values, viscous effects reduce, so it suggests a reduced amount of resistance to the fluid motion. In Figure 5, effects of the Eckert number are studied. The Eckert number relates the kinetic energy and heat enthalpy difference of the fluid flow. There is a direct link between the Eckert number and kinetic energy. So, rise in the Eckert number provides an increment in the kinetic energy; as a result, the thermal transport increases. 
TABLE 2: Skin friction values comparison for different magnetic field parameters and $\gamma=0$ and $A=0$, for $n=1$.

\begin{tabular}{lccc}
\hline$M$ & $\begin{array}{c}\text { Malik et al. [42] } \\
\text { Local similar }\end{array}$ & $\begin{array}{c}\text { Akbar et al. [44] } \\
\text { Local similar }\end{array}$ & $\begin{array}{c}\text { Present results } \\
\text { Nonsimilar }\end{array}$ \\
\hline 0 & -1 & -1 & -0.9585 \\
0.5 & -1.11802 & -1.11803 & -1.1780 \\
1 & -1.41419 & -1.41421 & -1.3705 \\
5 & -2.44945 & -2.44949 & -2.4393 \\
10 & -3.31657 & -3.31663 & -3.3293 \\
100 & -10.04981 & -10.04988 & -10.181 \\
500 & -22.38294 & -22.38303 & -22.6960 \\
1000 & -31.63851 & -31.63859 & -32.0855 \\
\hline
\end{tabular}

TABle 3: Friction coefficient for $A, \gamma$, and $M$ for $n=1$.

\begin{tabular}{llcc}
\hline$\gamma$ & $A$ & $M$ & $(1 / 2) \mathbf{R e}_{\mathbf{b}}^{1 / \mathbf{n}+1} \mathbf{C}_{\mathbf{f}}$ \\
\hline 0 & 1 & 0.3 & -1.6425 \\
0.25 & & & -2.0295 \\
0.5 & & & -2.4598 \\
0.75 & 1 & -2.9276 & -2.3628 \\
0.4 & 2 & & -2.8125 \\
& 3 & -3.2765 & -3.7400 \\
& 4 & 0.25 & -1.8377 \\
& 1 & 0.5 & -1.9792 \\
& & 0.75 & -2.1138 \\
& & & -2.2422 \\
\hline
\end{tabular}

TABLE 4: Nusselt number for $\gamma, \operatorname{Pr}$, and Ec for $n=1$.

\begin{tabular}{lccc}
\hline$\gamma$ & $\operatorname{Pr}$ & $\mathrm{Ec}$ & $\mathbf{R e}_{\mathbf{b}}^{-(-1 / \mathbf{n}+1)} \mathbf{C}_{\mathbf{f}}$ \\
\hline 0 & 1 & 0.1 & 0.5588 \\
0.25 & & & 0.6575 \\
0.5 & & & 0.7213 \\
0.75 & 1 & & 0.7656 \\
0.4 & 2 & 0.7069 & 0.9167 \\
& 3 & 0 & 1.0910 \\
& 4 & 0.25 & 1.2400 \\
& 1 & 0.5 & 0.7933 \\
& & 0.75 & 0.7391 \\
& & 0.6849 \\
\hline
\end{tabular}

Figure 6 displays the effect of the curvature parameter on thermal distribution. The enlarged curvature parameter contracts the cylinder's radius, so as an outcome, velocity as well as the kinetic energy which boosts the thermal transport enhances. Figure 7 assess the behavior of Prandtl number; it relates fluid viscosity with the thermal conductivity.
Therefore, it evaluates the relation between momentum and the thermal transport capacity of the fluid. Enhancement in the Prandtl number reduces the thermal dissipation and the temperature distribution falls due to the weak heat transfer rate. This shows that strengthening the Prandtl number plunges down the temperature distribution. 


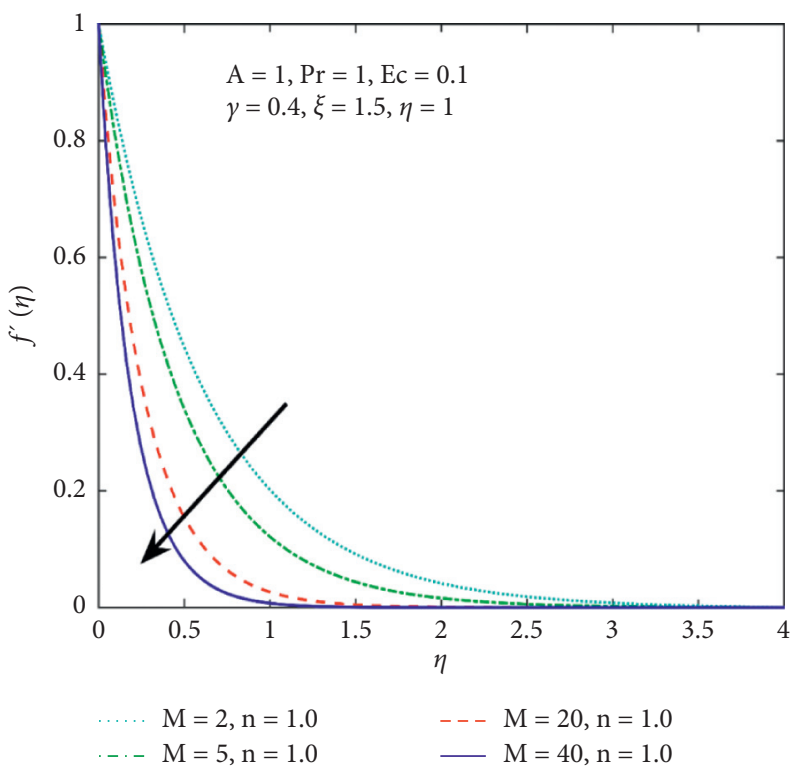

Figure 2: Velocity profile $f^{\prime}(\eta)$ for different values of magnetic parameter $(M)$.

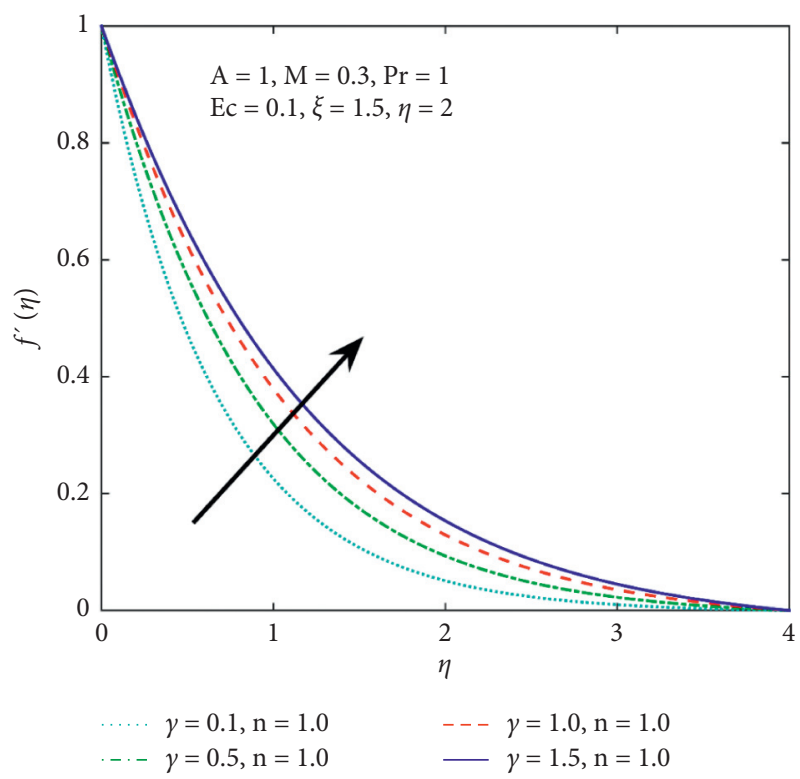

Figure 3: Velocity profile $f^{\prime}(\eta)$ for different values of curvature parameter $(\gamma)$. 


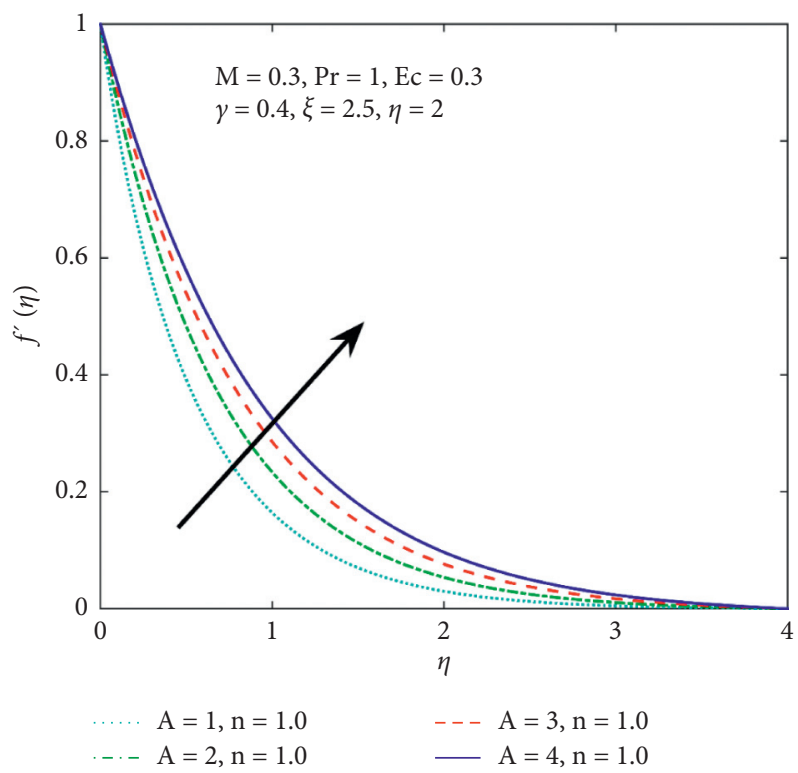

FIgURE 4: Velocity profile $f^{\prime}(\eta)$ for different values of material parameter $(A)$.

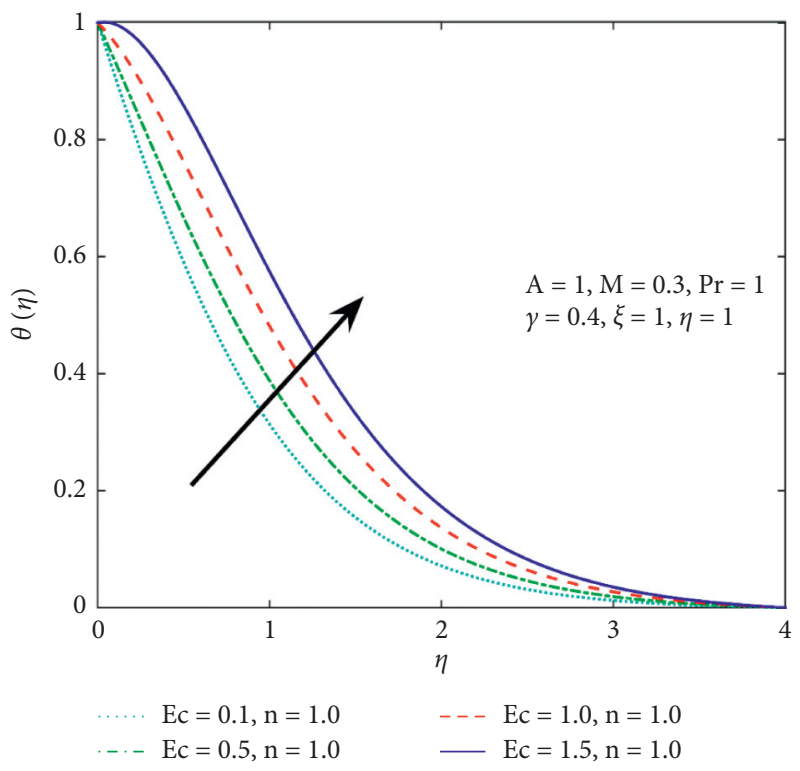

Figure 5: Thermal profile $\theta(\eta)$ for different values of Eckert number $(E c)$. 


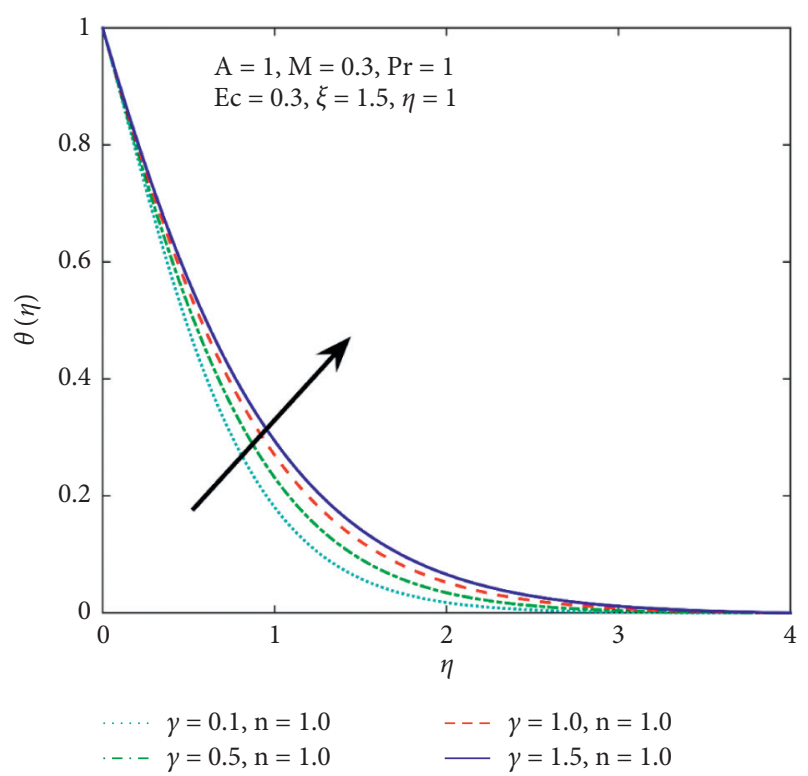

Figure 6: Thermal profile $\theta(\eta)$ for different values of curvature parameters $(\gamma)$.

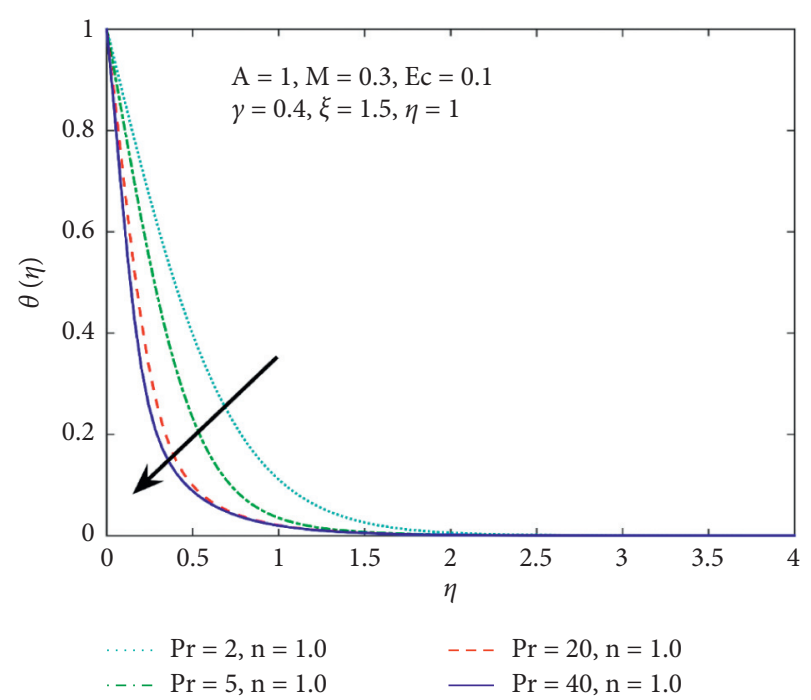

Figure 7: Thermal profile $\theta(\eta)$ for different values of Prandtl numbers (Pr).

\section{Conclusion}

In this study, the BL flow of Sisko fluid across a stretching cylinder with the impacts of viscous dissipation and the magnetic field is evaluated numerically. The numerical simulations of the nonsimilar model show that the rise in material parameter decays the viscous forces and, in result, enhances the flow rate, and the increment in the curvature parameter lift flow rate, while the augmentation in the magnetic field parameter, enhancing the resistance force which diminishes the velocity profile. Rise in dimensionless curvature and viscous dissipation parameters raises the thermal profile of the fluid. An increment in the Prandtl number decreases the thermal dissipation which declines the temperature distribution because of low heat transfer rate. Rising values of the Eckert number upsurges the temperature distribution profile. Also, increasing estimations of material and curvature parameters enhances the temperature profile. Friction forces extend for greater values of $A, M$, and $\gamma$. Increment in $\gamma, \operatorname{Pr}$, and $n$ gives enhancement of the Nusselt number.

\section{Data Availability}

The data that support the findings of this study are available from the corresponding author upon reasonable request.

\section{Conflicts of Interest}

The authors declare that they have no conflicts of interest.

\section{Acknowledgments}

The authors extend their appreciation to the deputyship for Research and Innovation, Ministry of Education in Saudi Arabia, to fund this research work through the project no. IFP-2020-20.

\section{References}

[1] A. Al-Sharify, T. Zainab, L. M. A. Faisal, T. A. Al-Sharif, N. T. Al-Sharify, and F. M. A. Faisal, "Removal of analgesic paracetamol from wastewater using dried olive stone," International Journal of Mechanical Engineering \& Technology, vol. 9, no. 13, pp. 293-299, 2018.

[2] A. Al-Qaisi, Q. Maryam, L. Faisal, Z. T. Al-Sharify, and T. A. Al-Sharify, "Possibility of utilizing from lemon peel as a sorbent in removing of contaminant such as copper ions from simulated aqueous solution," International Journal of Civil Engineering \& Technology, vol. 9, pp. 571-579, 2018.

[3] I. Cohen and D. Weihs, "Rheology and microrheology of natural and reduced-calorie Israeli honeys as a model for high-viscosity Newtonian liquids," Journal of Food Engineering, vol. 100, no. 2, pp. 366-371, 2010.

[4] A. W. Sisko, "The flow of lubricating greases," Industrial \& Engineering Chemistry, vol. 50, no. 12, pp. 1789-1792, 1958.

[5] F. T. Akyildiz and K. Vajravelu, E. Sweet, R. N. Mohapatra, E. Sweet, and R. A. Van Gorder, Implicit differential equation arising in the steady flow of a Sisko fluid," Applied Mathematics and Computation, vol. 210, no. 1, pp. 189-196, 2009.

[6] S. Nadeem and N. S. Akbar, "Peristaltic flow of Sisko fluid in a uniform inclined tube," Acta Mechanica Sinica, vol. 26, no. 5, pp. 675-683, 2010.

[7] M. Khan, S. Munawar, and S. Abbasbandy, "Steady flow and heat transfer of a Sisko fluid in annular pipe," International Journal of Heat and Mass Transfer, vol. 53, no. 7-8, pp. 1290-1297, 2010.

[8] A. Munir, A. Shahzad, and M. Khan, "Convective flow of Sisko fluid over a bidirectional stretching surface," PLoS One, vol. 10, no. 6, Article ID e0130342, 2015.

[9] M. Y. Malik, T. S. Arif Hussain, and M. Awais, "Numerical solution of MHD Sisko fluid over a stretching cylinder and heat transfer analysis," International Journal of Numerical Methods for Heat \& Fluid Flow, vol. 26, 2016.

[10] S. Nadeem, R. Ul Haq, and C. Lee, "MHD flow of a Casson fluid over an exponentially shrinking sheet," Scientia Iranica, vol. 19, no. 6, pp. 1550-1553, 2012. 
[11] M. Mustafa and J. A. Khan, "Model for flow of Casson nanofluid past a non-linearly stretching sheet considering magnetic field effects," AIP Advances, vol. 5, no. 7, Article ID 077148, 2015.

[12] U. Farooq, D. Lu, S. Munir, M. Ramzan, M. Suleman, and S. Hussain, "MHD flow of Maxwell fluid with nanomaterials due to an exponentially stretching surface," Scientific Reports, vol. 9, no. 1, pp. 7312-7411, 2019.

[13] M. M. Rashidi, N. Kavyani, and S. Abelman, "Investigation of entropy generation in MHD and slip flow over a rotating porous disk with variable properties," International Journal of Heat and Mass Transfer, vol. 70, pp. 892-917, 2014.

[14] M. Y. Malik, A. Hussain, S. B. Awais et al., "Flow of Sisko fluid over a stretching cylinder and heat transfer with viscous dissipation and variable thermal conductivity: a numerical study," AIP Advances, vol. 6, no. 4, Article ID 045118, 2016.

[15] W. A. Khan, "Numerical simulation of Chun-Hui He's iteration method with applications in engineering," International Journal of Numerical Methods for Heat \& Fluid Flow, 2021.

[16] W. A. Khan, "Numerical and simulation analysis comparison of hydraulic network problem base on higher-order efficiency approach," Alexandria Engineering Journal, vol. 60, no. 5, pp. 4889-4903, 2021.

[17] H. C. Brinkman, "Heat effects in capillary flow I," Applied Scientific Research, vol. 2, no. 1, pp. 120-124, 1951.

[18] M. Abd El-Aziz, "Unsteady mixed convection heat transfer along a vertical stretching surface with variable viscosity and viscous dissipation," Journal of the Egyptian Mathematical Society, vol. 22, no. 3, pp. 529-537, 2014.

[19] S. Saleem and S. Nadeem, "Theoretical analysis of slip flow on a rotating cone with viscous dissipation effects," Journal of Hydrodynamics, Ser. B, vol. 27, no. 4, pp. 616-623, 2015.

[20] S. Shateyi, F. Mabood, and G. Lorenzini, "Casson fluid flow: free convective heat and mass transfer over an unsteady permeable stretching surface considering viscous dissipation," Journal of Engineering and Thermophysics, vol. 26, no. 1, pp. 39-52, 2017.

[21] I. Pop and M. Sheremet, "Free convection in a square cavity filled with a Casson fluid under the effects of thermal radiation and viscous dissipation," International Journal of Numerical Methods for Heat \& Fluid Flow, vol. 27, 2017.

[22] H. Upreti, S. K. Rawat, and M. Kumar, "Radiation and nonuniform heat sink/source effects on 2D MHD flow of CNTs$\mathrm{H} 2 \mathrm{O}$ nanofluid over a flat porous plate," Multidiscipline Modeling in Materials and Structures, vol. 16, no. 4, 2019.

[23] G. Jithender Reddy, R. Srinivasa Raju, and J. Anand Rao, "Influence of viscous dissipation on unsteady MHD natural convective flow of Casson fluid over an oscillating vertical plate via FEM," Ain Shams Engineering Journal, vol. 9, no. 4, pp. 1907-1915, 2018.

[24] Z. Shah, L. B. McCash, A. Dawar, and E. Bonyah, "Entropy optimization in Darcy-Forchheimer MHD flow of water based copper and silver nanofluids with Joule heating and viscous dissipation effects," AIP Advances, vol. 10, no. 6, Article ID 065137, 2020.

[25] D. Gopal, S. Saleem, S. Jagadha, F. Ahmad, A. Othman Almatroud, and N. Kishan, "Numerical analysis of higher order chemical reaction on electrically MHD nanofluid under influence of viscous dissipation," Alexandria Engineering Journal, vol. 60, no. 1, pp. 1861-1871, 2021.

[26] L. A. Lund, Z. Omar, J. Raza, and I. Khan, "Magnetohydrodynamic flow of $\mathrm{Cu}-\mathrm{Fe} 3 \mathrm{O} 4 / \mathrm{H} 2 \mathrm{O}$ hybrid nanofluid with effect of viscous dissipation: dual similarity solutions," Journal of Thermal Analysis and Calorimetry, vol. 143, no. 2, pp. 915-927, 2021.

[27] E. M. Sparrow and H. S. Yu, "Local non-similarity thermal boundary-layer solutions," Journal of Heat Transfer, vol. 93, no. 4, pp. 328-334, 1971.

[28] A. S. Rao, V. Ramachandra Prasad, N. Nagendra, N. B. Reddy, and O. Anwar Beg, "Non-similar computational solution for boundary layer flows of non-Newtonian fluid from an inclined plate with thermal slip," Journal of Applied Fluid Mechanics, vol. 92 pages, 2016.

[29] V. R. Prasad and B. Rushi Kumar, "Non-similar comutational solutions for double-diffusive MHD transport phenomena for non-newtnian nanofluid from a horizontal circular cylinder," Nonlinear Engineering, vol. 8, no. 1, pp. 470-485, 2019.

[30] H. Sardar, M. Khan, and L. Ahmad, "Local non-similar solutions of convective flow of Carreau fluid in the presence of MHD and radiative heat transfer," Journal of the Brazilian Society of Mechanical Sciences and Engineering, vol. 41, no. 2, p. 69, 2019.

[31] A. Bisht and R. Sharma, "Non-similar solution of Casson nanofluid with variable viscosity and variable thermal conductivity," International Journal of Numerical Methods for Heat \& Fluid Flow, 2019, inpress.

[32] U. Farooq, M. A. Ijaz, M. I. Khan, S. S. P. M. Isa, and D. C. Lu, "Modeling and non-similar analysis for Darcy-ForchheimerBrinkman model of Casson fluid in a porous media," International Communications in Heat and Mass Transfer, vol. 119, Article ID 104955, 2020.

[33] A. Raees, U. Farooq, M. Hussain, W. A. Khan, W. A. Khan, and F. B. Farooq, "Non-similar mixed convection analysis for magnetic flow of second-grade nanofluid over a vertically stretching sheet," Communications in Theoretical Physics, vol. 73, no. 6, Article ID 065801, 2021.

[34] U. Farooq, M. Hussain, M. A. Ijaz, W. A. Khan, and F. Bashir Farooq, "Impact of non-similar modeling on Darcy-Forchheimer-Brinkman model for forced convection of Casson nano-fluid in non-Darcy porous media," International Communications in Heat and Mass Transfer, vol. 125, Article ID 105312, 2021.

[35] E. M. Sparrow, H. Quack, and C. J. Boerner, "Local nonsimilarity boundary-layer solutions," AIAA Journal, vol. 8, no. 11, pp. 1936-1942, 1970.

[36] S. Chen, J. Zhang, F. Meng, and D. Wang, "A Markov chain position prediction model based on multidimensional correction," Complexity, vol. 2021, Article ID 6677132, 8 pages, 2021.

[37] F. Meng, A. Pang, X. Dong, C. Han, and X. Sha, "Ho optimal performance design of an unstable plant under bode integral constraint," Complexity, vol. 2018, Article ID 4942906, 8 pages, 2018.

[38] P. Hu, L. Cao, J. Su, Q. Li, and Y. Li, "Distribution characteristics of salt-out particles in steam turbine stage," Energy, vol. 192, Article ID 116626, 2020.

[39] X. Li, Z.-Q. Dong, P. Yu et al., "Effect of self-assembly on fluorescence in magnetic multiphase flows and its application on the novel detection for COVID-19," Physics of Fluids, vol. 33, no. 4, Article ID 042004, 2021.

[40] X. Han, W. Zheng, B. Zhang, Y. Li, T. Du, and C. He, "Crop evapotranspiration prediction by considering dynamic change of crop coefficient and the precipitation effect in backpropagation neural network model," Journal of Hydrology, vol. 596, Article ID 126104, 2021.

[41] P. Fan, R. Deng, J. Qiu, Z. Zhao, and S. Wu, "Well logging curve reconstruction based on kernel ridge regression," Arabian Journal of Geosciences, vol. 14, no. 16, pp. 1-10, 2021. 
[42] M. Y. Malik, A. Hussain, T. Salahuddin, and M. Awais, "Effects of viscous dissipation on MHD boundary layer flow of Sisko fluid over a stretching cylinder," AIP Advances, vol. 6, no. 3, Article ID 035009, 2016.

[43] R. R. Rangi and N. Ahmad, "Boundary layer flow past a stretching cylinder and heat transfer with variable thermal conductivity," Applied Mathematics, vol. 3, 2012.

[44] N. Sher Akbar, A. Ebaid, and Z. H. Khan, "Numerical analysis of magnetic field effects on Eyring-Powell fluid flow towards a stretching sheet," Journal of Magnetism and Magnetic Materials, vol. 382, pp. 355-358, 2015. 